\title{
Single Trial Discrimination between Right and Left Hand Movement-Related EEG Activity"
}

\author{
Sunyoung Cho ${ }^{1}$, Jung Ae Kim², Dong-Uk Hwang ${ }^{2}$, and Seung Kee Han ${ }^{1,2}$ \\ ${ }^{1}$ Basic Science Research Institute, Chungbuk National University, Cheongju, Korea, \\ sycho@chungbuk.ac.kr \\ ${ }^{2}$ Department of Physics, Chungbuk National University, Cheongju, Korea,
}

\begin{abstract}
We propose an EEG-based discrimination method for the right/left hand movement in a single trial. The EEG was recorded during the voluntary movement and imagination of the hand movement. We made a feature vector for every second that represents the characteristics to reflect the process of the right/left movement. It was composed of the ERD, ERS patterns of the mu and beta rhythm and the coefficients of the autoregressive model best fitting for the data of the given period. Linear discrimination of their distributions in the vector space classified the right/left hand movement-related EEG activity efficiently.
\end{abstract}

\section{Introduction}

The ongoing EEG (electroencephalogram) signals are including useful information to reflect the neuronal processing for the specific mental and/or physical functions. There is a plenty of evidence indicating the frequency-specific changes of EEG may correlate to the sensory, motor and cognitive processing $[1,2,3]$. With high temporal resolution and a low cost, EEG is widely used in assessing brain processes. This EEG signals could be applied for the communication between the brain and an electronic system like a computer - a Brain-Computer Interface (BCI) [4].

The EEG changes reflecting the human intention related the limb movements or the imagination of the movements have been researched extensively and applied to BCI $[5,6]$. During the preparation or imagination of the movements, the EEG signals show frequency-specific changes time-locked to the event. These event-related changes consist of decrease or increase of the power in given frequency bands, which might be due to decrease or increase in synchronous activities of the underlying neuronal populations. These are called event-related desynchronization (ERD) and eventrelated synchronization (ERS) respectively [7].

* This work was supported by Korean Research Foundation, KRF 2002-075-H0007 to S.Y. Cho, and a grant(M103KV010011 03K2201 01130) from Brain Research Center of the 21st Century Frontier Research Program funded by the Ministry of Science and Technology of Republic of Korea to S.K. Han. 
In this study, the EEG signals were recorded during the performance and imagination of the hand movement and analyzed to generate feature vectors for every second EEG data. A feature vector was composed of the ERD, ERS patterns of the mu and beta rhythm and the coefficients of the autoregressive model best fitting for the data of the given period. Linear discrimination of their distribution in the vector space divided the right and left hand movement efficiently.

\section{Method}

\subsection{EEG Data Acquisition}

Thirty-five subjects aged 19 to 25 years participated in the study. All subjects were right-handed and free of neurological disorders. The EEG was recorded from the whole scalp with $32 \mathrm{Ag} / \mathrm{AgCl}$ electrodes placed according to the international 10-20 system (Neuroscan amplifier, sampling rate $1000 \mathrm{~Hz}$, bandwidth filtering $1.5 \sim 100 \mathrm{~Hz}$ ). Three kinds of experimental paradigms were used; self-paced hand movement in which subjects push a button with the index finger on their own pace in 12-18 s intervals, tone-triggered hand movement in which subjects perform the movements after the presentation of tone $(1 \mathrm{kHz}$, duration $100 \mathrm{~ms})$, and tone-trigger imagination of hand movement in which subjects were instructed to imagine performing the movement after the tone stimulation. The EEG was recorded continuously to be selected $12 \mathrm{~s}$ epoch in each trial, time-locked with the movement-onset or tone stimulation.
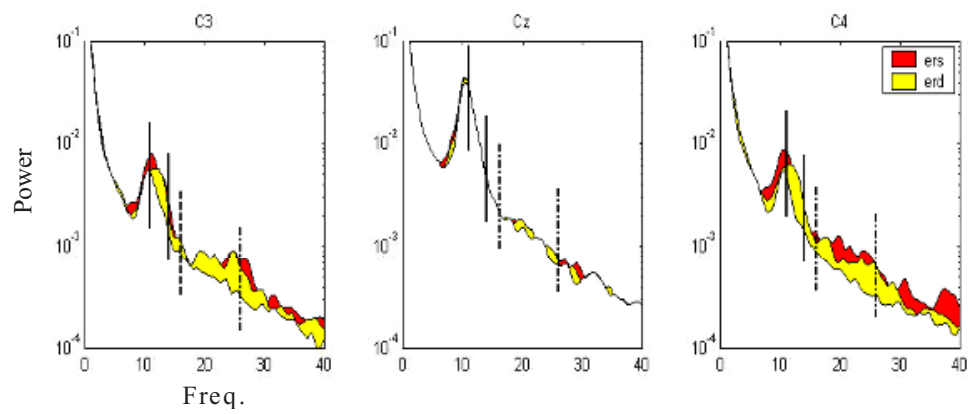

Fig. 1. Examples of $1 \mathrm{sec}$ power spectra from EEG data of $\mathrm{C} 3, \mathrm{Cz}$, and $\mathrm{C} 4$ recorded during premovement reference period (center line), movement period (lower line), and post-movement period (upper line) while tone-triggered left hand movement. The frequency ranges displaying significant power decrease or increase are marked with a pair of vertical solid lines of $11 \sim 14 \mathrm{~Hz}$ $(\mu)$ and a pair of dash-dot lines of $16 \sim 22 \mathrm{~Hz}(\beta)$ 


\subsection{Power Spectrum and ERD/ERS Computation}

To select the most reactive frequency components to reveal the ERD/ERS patterns related to the hand movement, the power spectra for three periods were compared. For the data from $\mathrm{C} 3, \mathrm{Cz}$, and $\mathrm{C} 4$ electrodes, the power spectra of 1-s pre-movement period as a reference, 1-s movement period around the movement onset, and 1-s postmovement period after movement offset were calculated. Examples from a subject are presented in Fig. 1. In these examples, similar to the formal studies [8, 9], they showed different in the frequency band between $11-14 \mathrm{~Hz}(\mathrm{mu})$ and $16-22 \mathrm{~Hz}$ (beta).

The ERD/ERS time curves were calculated for the selected frequency bands. This procedure involved band pass filtering, squaring of amplitude to obtain power values, averaging of power over all trials, normalizing, and computing of percentages with respect to the reference interval.

$$
E R D / E R S(\%)=\left(P_{\text {segment }}-P_{\text {reference }}\right) / P_{\text {reference }} \times 100
$$

\subsection{Coefficients of Autoregressive Model}

We adopted the coefficients of the autoregressive model as useful indices to discriminate right/left hand movement. In each trial, the EEG signals for 12-s epoch were divided into 1 -s window segments with $500 \mathrm{~ms}$ overlap. The coefficients of the autoregressive model best fitting for the data of each segment were calculated using the following model, in which delay time $\mathrm{d}=5$, and mode order $\mathrm{k}=6$.

$$
x_{n}=a_{1} x_{n-d}+a_{2} x_{n-2 d}+\cdots+a_{k} x_{n-k d}
$$

Fig. 2 illustrates the time curve of the one coefficient (a1) piled up across all trials. They showed the different patterns for the right/left directions (the left hand movement in this figure) and time locked to the movement onset and offset. Therefore, these coefficients were included in our feature vector.
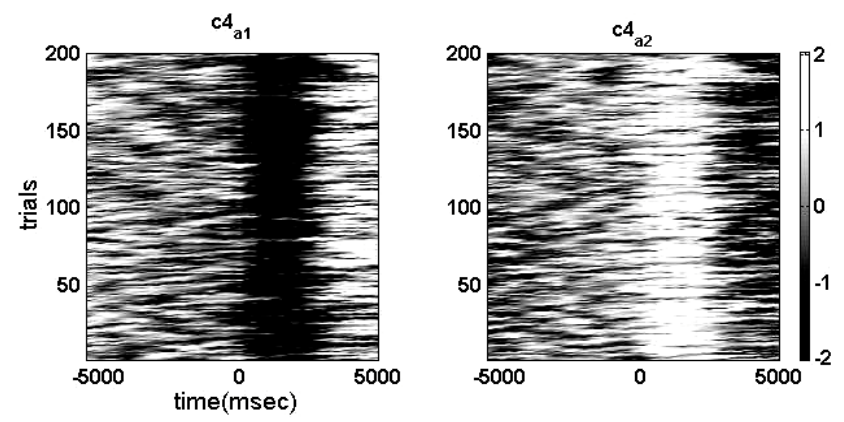

Fig. 2. The change of a coefficient of the autoregressive model in each trial while tonetriggered left hand movement. The $\mathrm{x}$ axis indicates the time (msec) from the movement onset, and $y$ axis indicates the trial number 


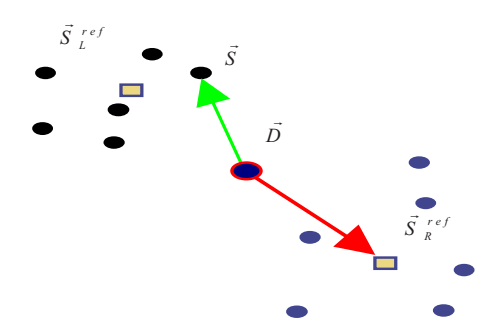

Fig. 3. Schematic diagram of the distribution of feature vectors in a vector space. If dark and thin circles represent the feature vectors of right and left movement, two rectangles $\left(\vec{s}_{L}^{\text {ref }}, \vec{s}_{R}^{\text {ref }}\right.$ ) indicate the middle points of each group. The center point $\vec{D}(t)$ is an average of two rectangles. The value of $\mathrm{d}(\mathrm{t})$ is inner product of $(\vec{s}(t)-\vec{D}(t))$ and $\left(\vec{s}_{L}^{r e J}(t)-\vec{D}(t)\right)$, which is qualifying the position of the vector compared to the middle point of one group, $\vec{s}_{L}^{r e J}(t)$ in this equation. If $\mathrm{d}(\mathrm{t})$ is positive value, therefore, it means the vector is included in the group. If negative, the vector is included in the other group

\subsection{Feature Vector and Liner Discrimination}

The feature vectors were composed with the characteristics proven to be useful for the right/left discrimination in our analysis. We made feature vectors for every 1 -s window segments using the EEG signals from $\mathrm{C} 3$ and $\mathrm{C} 4$ sites. A feature vector includes 6 coefficients of autoregressive model for $\mathrm{C} 3,6$ coefficients for $\mathrm{C} 4$, and the ratios of the power change in mu and beta bands for $\mathrm{C} 3$ and $\mathrm{C} 4$ (that is, ERD/ERS ratio in the period). To compare across trials, the values were standardized in each trial to the reference period of the formal $6 \mathrm{~s}$ before the tone onset.

$$
\begin{gathered}
\vec{S}(t)= \\
\left(\bar{a}_{1}^{C 3}, \bar{a}_{2}^{C 3}, \ldots, \bar{a}_{6}^{C 3}, \bar{a}_{1}^{C 4}, \bar{a}_{2}^{C 4}, \ldots, \bar{a}_{6}^{C 4}, \bar{P}_{h i-\alpha}^{C 3}, \bar{P}_{\beta}^{C 3}, \bar{P}_{h i-\alpha}^{C 4}, \bar{P}_{\beta}^{C 4}\right) \\
\bar{x}=\frac{x-\langle x\rangle_{\text {ref }}}{\sigma(x)_{\text {ref }}}
\end{gathered}
$$

Feature vectors of every window segments were projected to the vector space (16 dimensions in this case) for their distributions to be discriminated linearly. Fig 3 explained the definition of $\mathrm{d}(\mathrm{t})$, quantified the position of each vector in the vector space.

$$
\begin{aligned}
& \vec{S}_{L / R}^{r e f}(t)=\frac{1}{N_{L / R}} \sum \vec{S}_{L / R}(t) \\
& \vec{D}(t)=\left(\vec{S}_{L}^{e f}(t)+\vec{S}_{R}^{r e f}(t)\right) / 2 \\
& d(t)=(\vec{S}(t)-\vec{D}(t)) \cdot\left(\vec{S}_{R}^{r e f}(t)-\vec{D}(t)\right) \\
& \text { if } d>0 \rightarrow \text { Right } \\
& \text { if } d<0 \rightarrow \text { Lelf }
\end{aligned}
$$



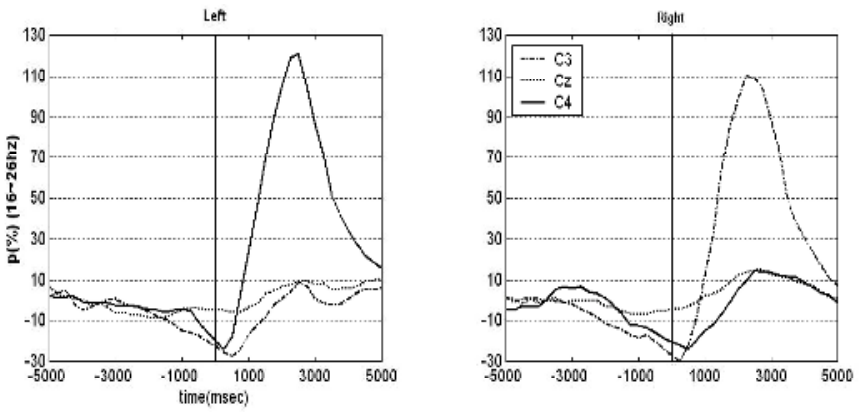

Fig. 4. Grand average time courses of $\beta$ rhythm in $\mathrm{C} 3$ (dash-dot line) $\mathrm{Cz}$ (thin line), and $\mathrm{C} 4$ (solid line) while tone-triggered movement of left (left box) and right (right box) hand. The $\mathrm{x}$ axis indicates the time (msec) from the onset of the tone (the vertical bar). The y axis indicates the percentage of the relative power change, to show ERD and ERS specifically dominant in the contralateral somatomotor area

\section{Results}

Fig. 4 displays the grand average ERD/ERS time courses of the beta band activity from $\mathrm{C} 3, \mathrm{Cz}$, and $\mathrm{C} 4$ data. For each side movement, it is seen that the post-movement power increases (ERS) are larger in the contralateral hemisphere than the ipsilateral hemisphere. In case of the mu band, a prominent ERD was found in the contralateral hemisphere, followed by ERS.
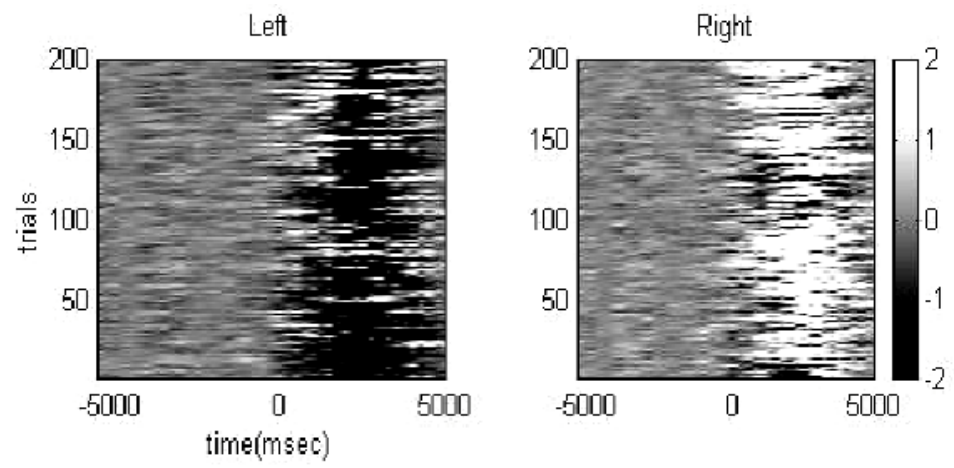

Fig. 5. The time course of $d(t)$ value of the feature vector while tone-triggered movement of left (left box) and right (right box) hand. The $\mathrm{x}$ axis indicates the time (msec) from the movement onset, and $y$ axis indicates the trial number 


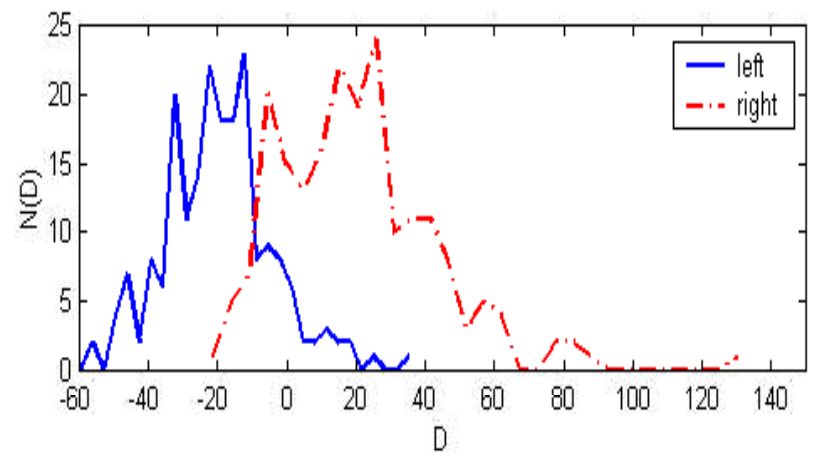

Fig. 6. Histogram of $\mathrm{d}(\mathrm{t})$ value of the feature vector while tone-triggered movement of left (solid line) and right (dot line) hand. The $\mathrm{x}$ axis indicates the value of $\mathrm{d}(\mathrm{t})$ and the $\mathrm{y}$ axis indicates the number of the feature vectors that have the value. In this subject, the recognition rates of left and right are $91 \%$ and $87 \%$

Fig. 5 illustrates the time curve of the $d(t)$ value piled up across all trials. They are changed consistently after movement onset by the right/left movement across the trials. We made a histogram accumulated by the value of $d(t)$ for every right/left trials in each subject. As shown in Fig. 6, the distributions for the right and left movement could be discriminated well. Table 1 presents the recognition ratios for right/left movement using the linear discrimination of the feature vectors for 6 subjects.

Table 1. The recognition rate while tone-triggered hand movement

\begin{tabular}{cccc}
\hline subject & Left(\%) & Right $(\%)$ & Total $(\%)$ \\
\hline HMA & 82.0 & 68.0 & 75.0 \\
JJH & 81.0 & 76.5 & 78.7 \\
CSY & 75.5 & 66.5 & 71.0 \\
KSM & 91.0 & 80.0 & 85.5 \\
JWR & 71.5 & 79.3 & 75.5 \\
PMJ & 72.0 & 80.0 & 76.0 \\
\hline Total & 78.8 & 75.1 & 77.1 \\
\hline
\end{tabular}

\section{Discussion}

For the application to the BCI system, it is necessary that the EEG features related to the human intent were analyzed with the EEG signals in a single trial. The present study determined the features that could reveal the intention and performance of the right/left hand movement and proposed the discrimination method using the features in a single trial. 

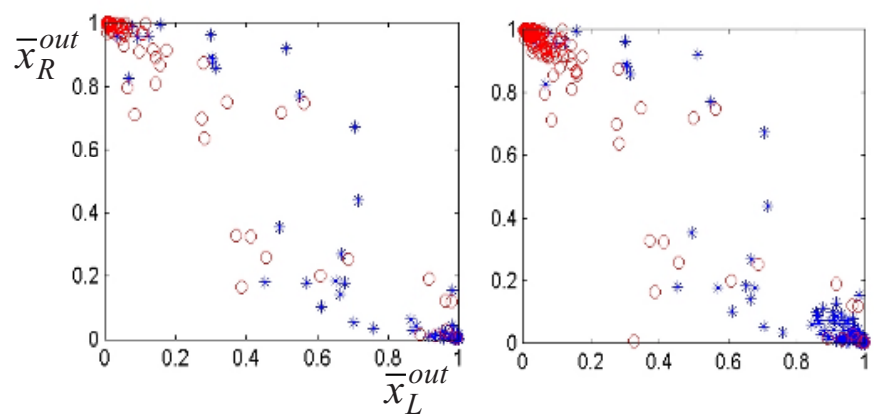

Fig. 7. The performance of the artificial neural network using the units of the feature vector as input nodes while tone-triggered hand movement. We set two output nodes for right and left, indicated in the $\mathrm{y}$ and $\mathrm{x}$ axis. Two kinds of test data set were applied to generate recognition rate $87 \%$ and $93 \%$

As units of our feature vector, we used the single-trail ERD/ERS patterns that were well known as grand averaged ones. And the coefficients of the autoregressive model were used as other units, which showed consistent time-course changes across the trials and differences in the right/left movement.

We further tried to use an artificial neural network for the discrimination of the right/left movement. The units of our feature vectors after the movement onset in each trial were used as the value of the input nodes (multi-layered perception model, input node 80 , one-layer hidden node 10 , output node 2 , and learning rule: feed-forward backpropagation). The preliminary result showed a similar recognition rate with the linear discrimination method (Fig. 7).

\section{References}

1. Bastiaansen, M.C.M., Bocker, K.B.E., Brunia, C.H.M., Munch, J.C., Spekreijse, H.: Eventrelated desynchronization during anticipatory attention for an upcoming stimulus: a comparative EEG/EMG study. Clin. Neurophysiol. 112 (2001) 393-403

2. Leocani, L., Toro, C., Zhuang, P., Gerlff, C. Hallett, M.: Event-related desynchronization in reaction time paradigms: a comparison with event-related potential and cortical excitability, Clin. Neurophysiol. 112 (2001) 923-930

3. Singer, W.: Synchronization of cortical activity and its putative role in informstion processing and learning, Annual Review of physiology 55 (1993) 349-374

4. Wolpaw, J. R., Birbaumer, N., McFarland, D.J., Pfurtscheller, G., Vaughan, T.M.: Braincomputer interfaces for communication and control, Clin. Neurophysiol. 113 (2002) 767 791

5. Pfurtscheller, G., Neuper, C., Guger, C., Harkam, W., Ramoser, H., Schlögl, A.,Obermaier, B., Pregenzer, M.: Current Trends in Graz Brain-Computer Interface (BCI) Research, IEEE Trans. Rehabil. Engineering 8 (2000) 216-219 
6. Babiloni, F., Cincotti, F., Bianchi, L., Pirri, G., Millan, J.R., Mourin, O.J., Salinari, S., Marciani, M.G.: Recognition of imagined hand movements with low resolution surface Laplacian and linear classifiers, Medical Engineering \& Physics 23 (2001) 323-328

7. Pfurtscheller, G., Lopes da Silva, F.H.: Event-related EEG/MEG synchronization and desynchronization : basic principles, Clin. Neurophysiol. 110 (1999) 1942-1857

8. Stancak, A.J., Riml, A., Pfurtscheller, G.: The effects of external load an movement-related changes of the sensorimotor EEG rhythms, Elec. and Clin. Neurophysiol. 102 (1997) 495504

9. Stancak, A., Pfurtscheller,G.: Event-related desynchronisation of central beta-rhythms during brisk and slow self-paced finger movements of dominant and nondominant hand, Cognitive Brain Research 4 (1996) 171-183 